\title{
Endometrial Cancer Arising in Adenomyosis That Could Not Be Diagnosed by Endometrial Biopsy: A Case Report
}

\author{
Jun Chikumi,* Tetsuro Oishi, *† Takaya Nakaso,* Mayumi Sawada,* Akiko Kudoh,* Hiroaki Komatsu,* Shinya Sato,* \\ Fuminori Taniguchi* and Tasuku Harada* \\ *Department of Obstetrics and Gynecology, School of Medicine, Faculty of Medicine, Tottori University, Yonago 683-8503, Japan, \\ †Department of Obstetrics and Gynecology, Matsue City Hospital, Matsue 690-8509, Japan
}

\section{ABSTRACT}

Uterine adenomyosis is an estrogen-dependent tumor and one of the most common benign diseases in sexually mature women. The frequency of endometrial cancer associated with adenomyosis has been reported to be $18 \%-66 \%$. On the other hand, endometrial cancer arising in adenomyosis (EC-AIA) is extremely rare. EC-AIA is now considered a different entity from and has a worse prognosis than endometrial cancer with adenomyosis (EC-A). In the present study, we report a case of endometrial cancer with adenomyosis in which endometrial biopsy failed to provide a definitive diagnosis. A 63-year-old female patient presented with endometrial thickening. Endometrial cytology was positive, and magnetic resonance imaging (MRI) showed small lesions suggestive of endometrial cancer with shallow invasion and adenomyosis. However, an endometrial biopsy showed only metaplasia, and careful follow-up was initiated. Subsequent endometrial cytology showed enlarged and round nuclei, uniform chromatin distribution, no thickening of nuclear margins, and abundant cytoplasm appearing in a sheet-like arrangement, suggesting atypical cells of endometrial glands with metaplasia. Three suspicious positive results and one positive result were observed, but repeated biopsies did not lead to the diagnosis of malignancy. The patient underwent diagnostic hysterectomy 19 months after the initial visit. The postoperative histopathological diagnosis was stage IA endometrial cancer (endometrioid carcinoma G1). This case of endometrial cancer associated with adenomyosis was difficult to diagnose. Our findings demonstrate that EC-AIA should be considered even if no lesions were detected by endometrial biopsy.

Key words adenomyosis; endometrial cancer; gyne-

Corresponding author: Tetsuro Oishi, $\mathrm{MD}, \mathrm{PhD}$

tetsuro@matsue-cityhospital.jp

Received 2021 November 24

Accepted 2021 December 24

Online published 2022 January 29

Abbreviations: ATEC, atypical endometrial cells; EC-A, endometrial cancer with adenomyosis; EC-AIA, endometrial cancer arising in adenomyosis; MRI, magnetic resonance imaging; TVUS, transvaginal ultrasound cologic oncology

Uterine adenomyosis is an estrogen-dependent tumor and one of the most common benign diseases in sexually mature women. The frequency of endometrial cancer associated with adenomyosis has been reported to be $18 \%-66 \% .{ }^{1}$ Endometrial cancer arising in adenomyosis (EC-AIA) and endometrial cancer with adenomyosis (EC-A) are two different entities; the former is considered to have a worse prognosis than the latter. ${ }^{2}$

EC-AIA is extremely rare. ${ }^{3}$ In a study of 2,080 endometrial cancer cases, the frequency of EC-AIA was $1.35 \%{ }^{4}$ EC-AIA is sometimes difficult to preoperatively diagnose. In the present study, we report a case of endometrial cancer with adenomyosis in which endometrial biopsy failed to provide a definitive diagnosis.

\section{PATIENT REPORT}

The patient was a 63-year-old woman (gravida 4, para 2) with a history of appendectomy at the age of 15 years. Her periods had ceased at the age of 50. No relevant family history was noted. She got cervical cancer screening at a gynecology clinic in June X. Cytology of her Pap test indicated "Other malignant neoplasms," and endometrial thickening was pointed out by transvaginal ultrasound (TVUS). Endometrial cytology showed atypical cell clusters with nuclear overlapping, glandular dilatation, and abnormal branching. She was referred as suspected endometrial cancer to our department in August X. TVUS showed a thickened endometrium of $13 \mathrm{~mm}$, but endometrial biopsy showed no obvious malignant findings. Pelvic contrast-enhanced MRI showed a mass with slight myometrial invasion in the uterine fundus, suggesting the presence of uterine cancer. Adenomyosis with cysts in the myometrium was also found (shown in Fig. 1). Tumor markers were as follows: CA125, 8.1 U/mL; CA19-9, 6.3 U/mL; and CEA, $1.0 \mathrm{ng} /$ $\mathrm{mL}$. Hysteroscopy showed endometrial fluffing with atypical blood vessels from the left side of the uterine fundus to the anterior wall. The histological examination of the endometrium by dilatation $\&$ curettage was performed, but no definitive diagnosis could be made (shown in Fig. 2). Endometrial cytology was performed 


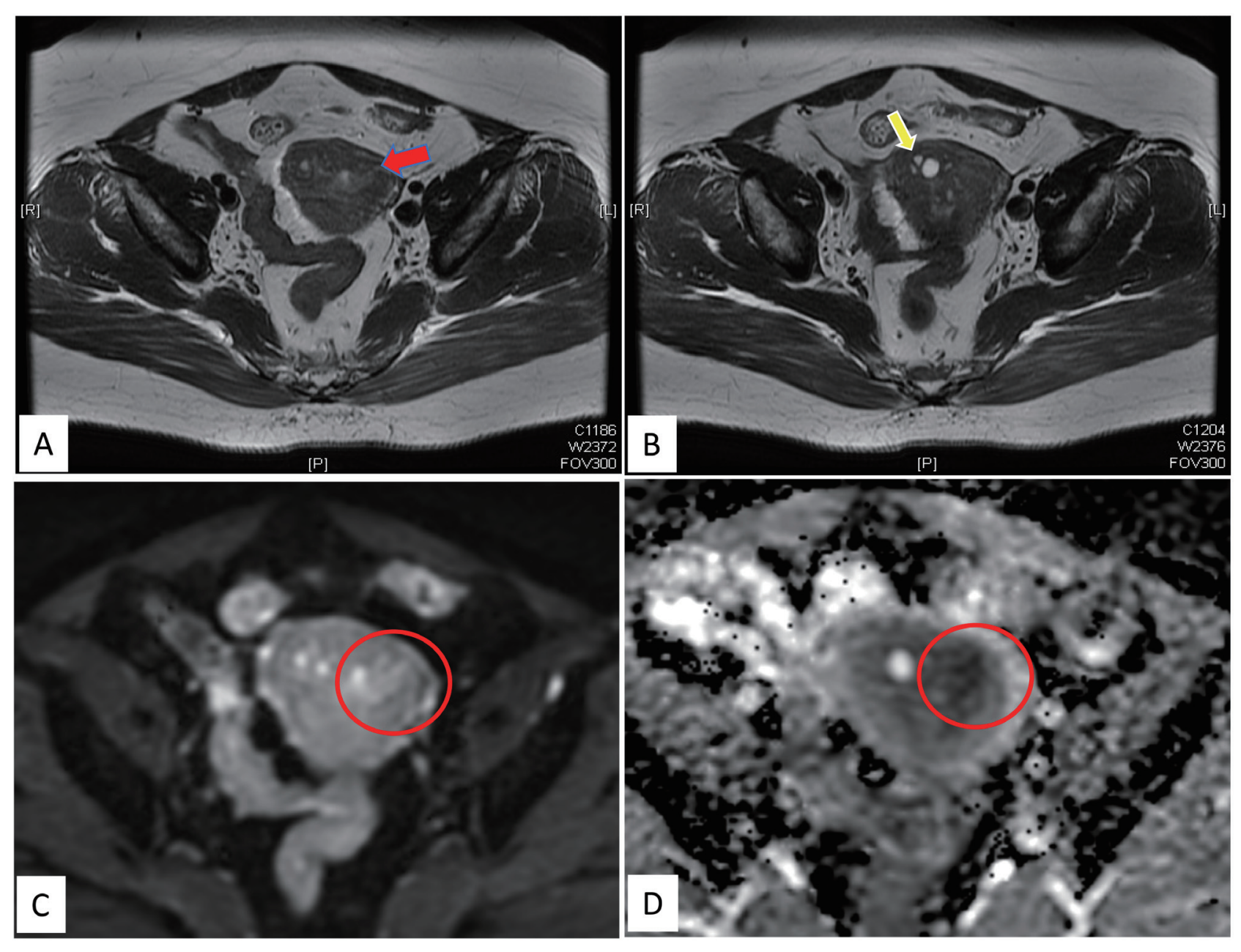

Fig. 1. Pelvic magnetic resonance imaging (MRI). A: T2-weighted sequence in axial orientation. Small lesion with hyper-equal intense signal suggesting shallow invasion (red arrow). B: T2-weighted sequence in axial orientation. Hyperintense locus surrounded by small cystic lesions suggesting adenomyosis (yellow arrow). C: A high signal on diffusion-weighted images suggesting uterine cancer lesion (red circle). D: Low apparent diffusion coefficient suggesting uterine cancer lesion (red circle).

every 3-6 months. If the cytology was suspicious or positive, endometrial biopsy was performed. However, five rounds of biopsy did not produce a definitive diagnosis. In January of $\mathrm{X}+2$, a diagnostic hysterectomy and bilateral adenectomy were performed (shown in Figs. 3A and B). The postoperative histopathological diagnosis was stage 1A endometrial cancer (pTlaNxM0) (shown in Figs. 3C-F). The patient has no evidence of disease 22 months after the surgery.

\section{DISCUSSION}

The currently proposed pathological diagnostic criteria for EC-AIA are as follows: (1) absence of tumor in the superficial layer of the endometrium and other pelvic areas, (2) tumor originating from the epithelium of the adenomyotic area and no invasion from other sites, and (3) abnormal adenomyotic glandular ducts surrounded by endometrial stromal cells. ${ }^{5}$ EC-A is defined as endometrial cancer coexisting with adenomyosis, whose endometrial glands and stroma in the myometrium are away from the endometrial junction. Although EC-AIA is extremely rare, the actual number of EC-AIA may be higher than reported because the exposure of the lesion to the lumen of the uterus with progression would fall outside the diagnostic criteria. Novak et al. ${ }^{6}$ reported that the originating site is difficult to histologically prove in advanced EC-A. In the present case, part of the lesion was also located in the superficial layer of the endometrium. However, the lesion in the myometrium was the main locus, strongly suggesting the possibility of EC-AIA.

EC-AIA initially arises within adenomyotic epithelium in the myometrial layer but easily reaches the myometrial stroma due to the lack of anatomical 

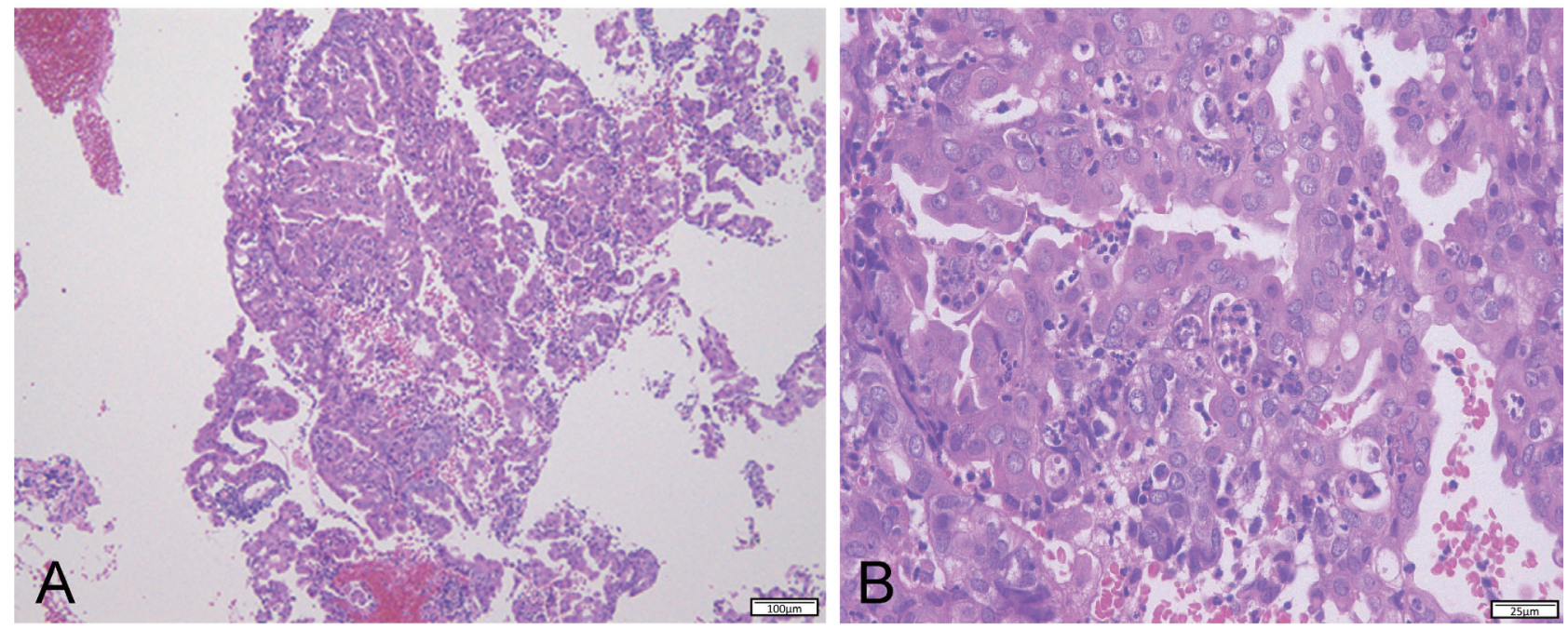

Fig. 2. A, B) Histological findings of endometrial biopsy (hematoxylin and eosin staining). A, Bar $=100 \mu \mathrm{m} . \mathbf{B}, \mathrm{Bar}=25 \mu \mathrm{m}$. Similar to the atypical endometrial cells-undetermined significance (ATEC-US) images, papillary growth of atypical epithelial cells with rounded and enlarged nuclei and large sporangia were observed, accompanied by a high degree of neutrophilic infiltration. These features suggest eosinophilic or papillary metaplasia.

barrier in the basal layer of the endometrium. Cancer that invade directly into the uterine stroma can easily metastasize to the lymphatic and vascular systems. This can partly explain the poor prognosis of EC-AIA.

Although MRI is a useful modality in the diagnosis of uterine cancer, the characteristic findings in EC-AIA have not been established. ${ }^{7}$ In this case, the lesion showed hyper-equal signals to the myometrium on T2weighted images, a high signal on diffusion-weighted images, low apparent diffusion coefficient, weaker contrast effect than the myometrium on dynamic phase 1 , and slightly heterogeneous contrast effect on the delayed phase. In the delayed phase, the contrast effect was somewhat heterogeneous. Based on the initial MRI, this area was strongly suspected to be uterine cancer with mild myometrial invasion, which was one of the reasons for the hysterectomy without diagnosis of malignancy. Additionally, adenomyosis was also noted, and the possibility of invasion along the adenomyosis was pointed out. An MRI scan showed similar findings 14 months later but with an enlargement of the area and clarification of the findings over time, making it more suspiciously a neoplastic lesion.

EC-AIA generally lacks clinical symptoms, and because the lesion is located in the muscle layer, obtaining clear findings on various tests, such as cytology, histology, and MRI, is difficult. As a result, diagnosis is often delayed. ${ }^{8}$ This is thought to be another reason for the poor prognosis of EC-AIA. In the present case, although the endometrial cytology was positive or suspiciously positive, the biopsy tissue showed only metaplastic tissue, and it took 19 months from the initial diagnosis to the final definitive diagnosis. There was an option of hysterectomy at an earlier stage, but this delay resulted from determining the amount of invasiveness. In addition, a hysterectomy and bilateral salpingo-oophorectomy, retroperitoneal lymph node dissection, intra-abdominal retrieval, and, if necessary, omentectomy are originally performed as standard procedures for uterine endometrial cancer. Accordingly, the preoperative definitive diagnosis of uterine endometrial cancer is greatly related to the surgical procedure and is therefore important. As a result, we needed a followup period until the lesion showed a slight increase on MRI. The fact that the lesion suspected by the MRI scan was small and equivalent to stage I cancer was also a significant factor in the follow-up. During this period, we explained the above information to the patient sufficiently, and conducted careful follow-up.

We speculate that one reason for the failure to make a definitive diagnosis of cancer was the sampling error caused by the fact that the lesion was mainly located in the myometrium. The other reason may be that the endometrial epithelium had metaplastic changes in the superficial layer of the carcinoma, interfering with the diagnosis. Kaku et al. ${ }^{9}, 10$ reported that $45 \%$ of normal endometria, $70 \%$ of endometrial proliferative diseases, and $60 \%$ of peri-cancerous endometrium were found to have metaplasia. Although metaplasia itself does not indicate cancer, if it persists, malignancy should be suspected, which is an important finding in clinical practice. In the present case, immunostaining with 


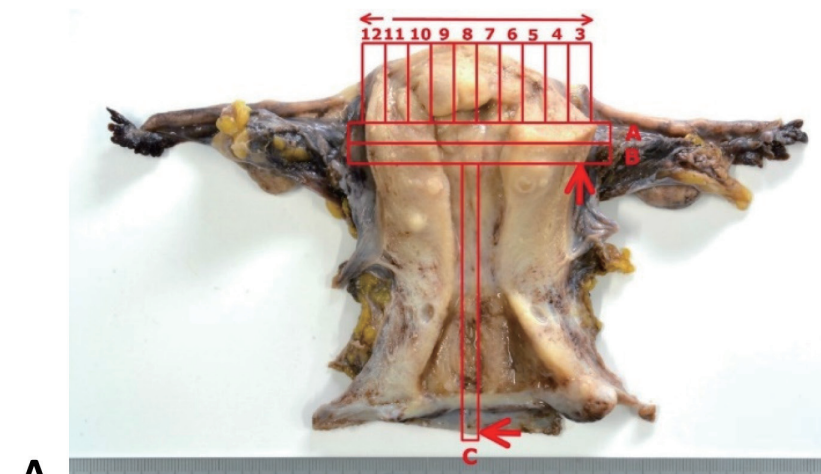

A
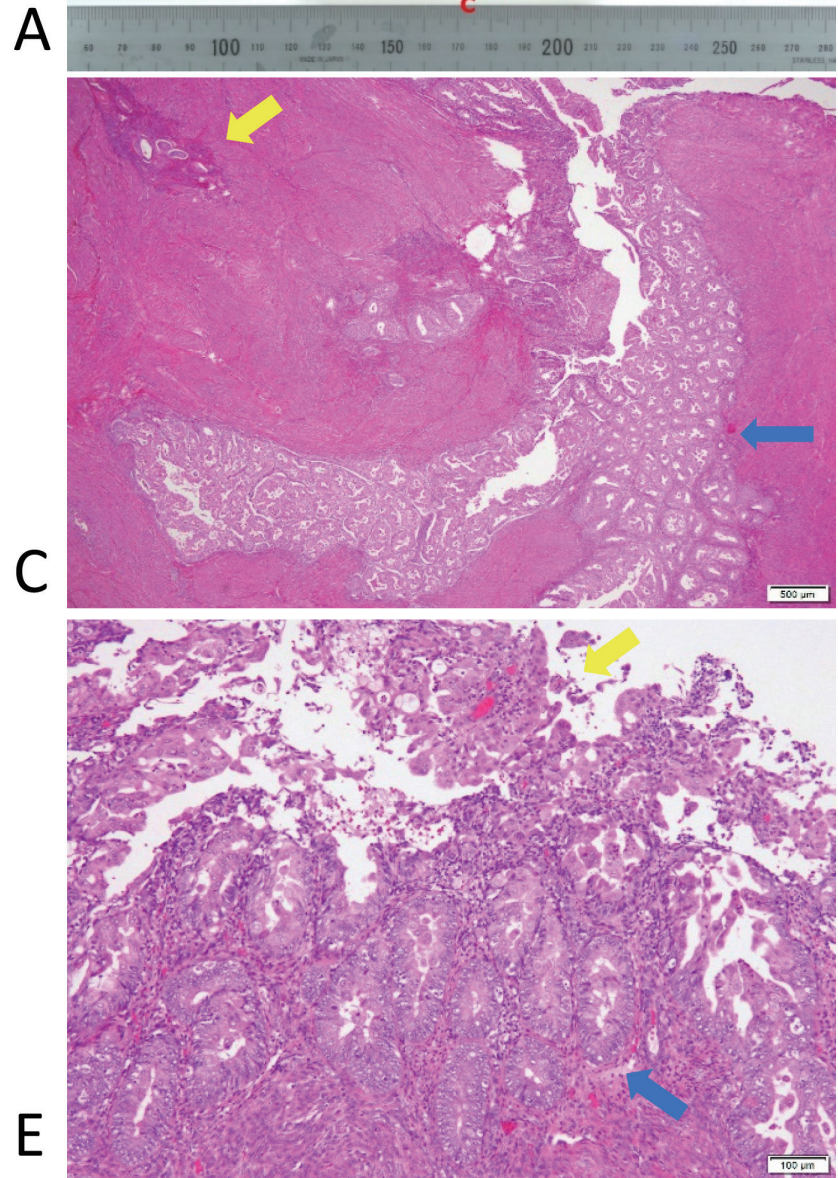

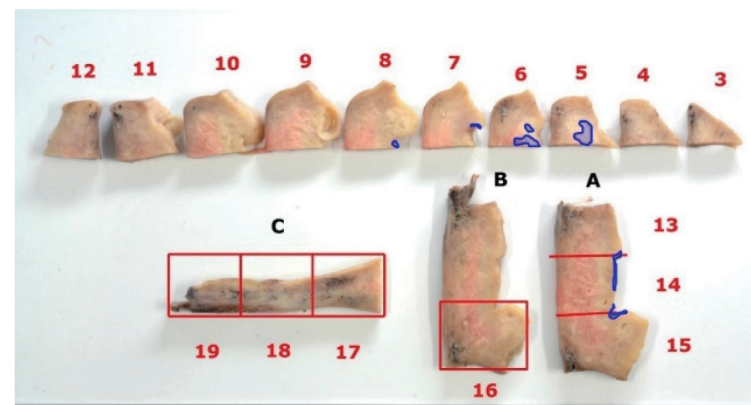

B
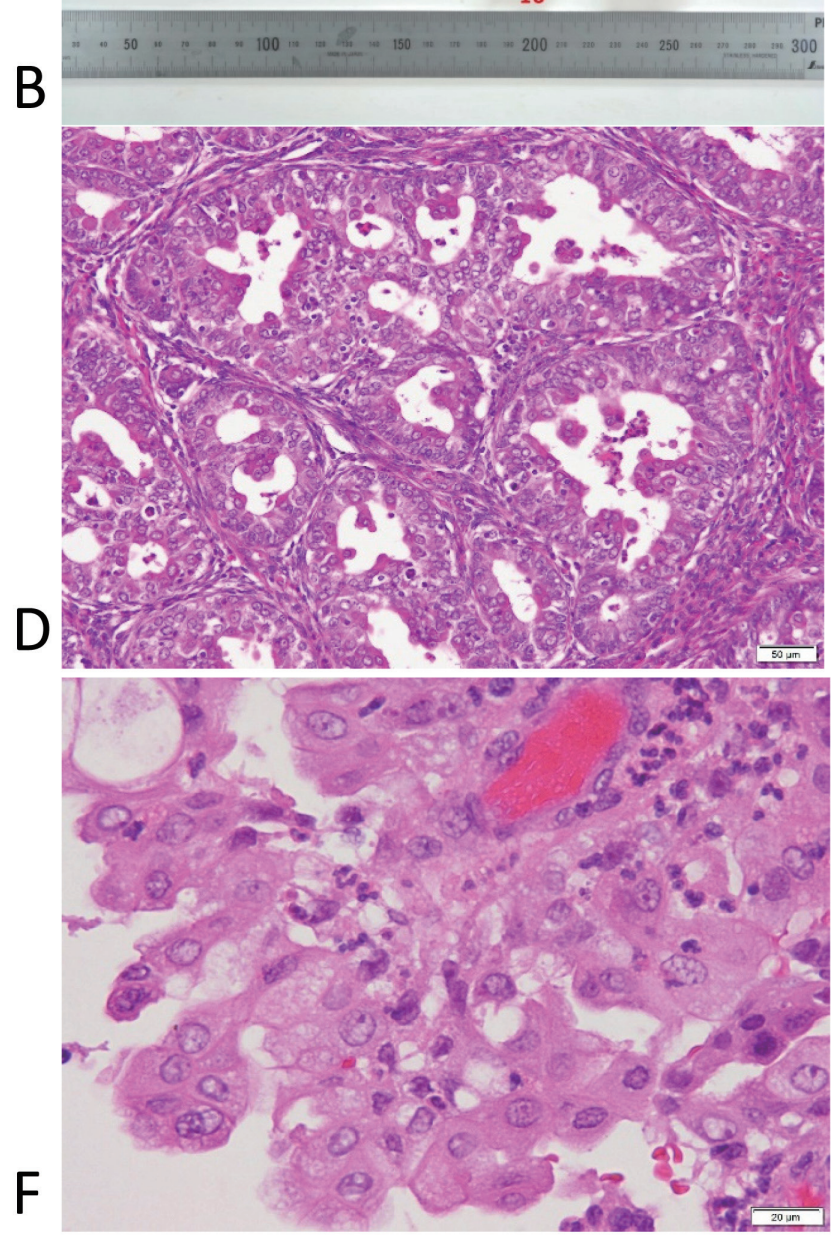

Fig. 3. A, B) Gross findings from hysterectomy specimens and blocking: A and B indicate horizontal sections of the uterine body and $\mathrm{C}$ indicates a sagittal section from the body to the cervix. Blocks 5-8 and 14 indicate cancer lesions found in the myometrium. C) Histologic findings indicating a tumor (invasive front of adenocarcinoma). Adenocarcinoma (blue arrow) and adenomyosis (yellow arrow), HE, Bar $=500 \mu \mathrm{m}$. D) Endometrial cancer was localized in adenomyosis, HE, Bar $=50 \mu \mathrm{m}$. The tumor was localized in the myometrium rather than in the endometrial surface of the uterus. The tumor was an endometrioid carcinoma G1, which mainly grew in the adenomyosis of the uterus, with extensive adenomyotic tissue in the background. The border between the tumor and the myometrium was clear, and no direct invasion into the myometrium was seen. E) Histologic findings indicating a tumor (superficial lesion). Endometrioid adenocarcinoma (blue arrow) and metaplasia (yellow arrow), HE, Bar $=100 \mu \mathrm{m} . \mathbf{F}$ ) Metaplasia, HE, Bar $=20 \mu \mathrm{m} . \mathrm{The}$ superficial intima of the tumor showed metaplastic tissue.

beta-catenin, MIB-1, and p53 was also performed on the biopsied tissue, but none of the findings immediately suggested malignancy such as endometrial cancer. It is hoped that diagnostic biomarkers can be established for cases with metaplastic changes or atypical endometrial cells (ATEC).

In general, no report suggests the benefit of adjuvant chemotherapy or radiotherapy in low-risk patients for postoperative recurrence with uterine cancer. Therefore, in principle, we do not perform adjuvant 
therapy in low-risk patients with endometrial cancer. The pathological evaluation of myometrial invasion is complicated because the pathogenesis of this disease is different from that of typical endometrial cancer. In this case, the border between the tumor and the myometrium was clear and no stromal invasion into the myometrium was seen. We finally made a diagnosis of no myometrial invasion based on this information. Although the disease generally has a poor prognosis, we judged this case to be in the low-risk group for postoperative recurrence and did not perform postoperative adjuvant therapy.

The therapeutic significance of adding lymph node dissection at reoperation is not clear when lymph node dissection has not been performed in patients with postoperative endometrial cancer, as in this case. The frequency of lymph node metastasis in endometrial cancer corresponding to the low-risk group for postoperative recurrence is low at about $3 \%$. Therefore, the national guideline suggests that careful follow-up can be performed without additional dissection for patients who are presumed to be at low risk for postoperative recurrence, as in this case, and we support this.

We reported a case of endometrial cancer complicated by adenomyosis of the uterus that eluded definitive diagnosis through the usual diagnostic methods. EC-AIA is not yet a well-established disease concept, and decisive diagnostic methods and characteristic imaging findings have not been established. In addition, many problems arise in pathological diagnosis, such as the lack of clear criteria for evaluating muscle layer invasion. Even if no lesion is detected by endometrial tissue biopsy, if the background suggests the presence of adenomyosis, further detailed examination needs to be conducted with the consideration of EC-AIA.

Consent for publication: Informed consent was obtained from the patient for publication of the details of their medical case and any accompanying images.

The authors declare no conflict of interest.

\section{REFERENCES}

1 Vercellini P, Viganò P, Somigliana E, Daguati R, Abbiati A, Fedele L. Adenomyosis: epidemiological factors. Best Pract Res Clin Obstet Gynaecol. 2006;20:465-77. DOI: 10.1016/ j.bpobgyn.2006.01.017, PMID: 16563868

2 Matsuo K, Cahoon SS, Gualtieri M, Scannell CA, Jung $\mathrm{CE}$, Takano T, et al. Significance of adenomyosis on tumor progression and survival outcome of endometrial cancer. Ann Surg Oncol. 2014;21:4246-55. DOI: 10.1245/s10434-014-38806, PMID: 25001096

3 Machida H, Maeda M, Cahoon SS, Scannell CA, Garcia-Sayre J, Roman LD, et al. Endometrial cancer arising in adenomyosis versus endometrial cancer coexisting with adenomyosis: are these two different entities? Arch Gynecol Obstet. 2017;295:1459-68. DOI: 10.1007/s00404-017-4375-z, PMID: 28444512

4 Chao X, Wu M, Ma S, Tan X, Zhong S, Bi Y, et al. The clinicopathological characteristics and survival outcomes of endometrial carcinoma coexisting with or arising in adenomyosis: A pilot study. Sci Rep. 2020;10:5984. DOI: 10.1038/ s41598-020-63065-w, PMID: 32249826

5 Colman HI, Rosenthal AH. Carcinoma developing in areas of adenomyosis. Obstet Gynecol. 1959;14:342-8. PMID: 13811320

6 Novak ER. Novak's gynecologic and obstetric pathology. 8th edition. Philadelphia: W, B. Saunders Co; 1979. p. 280-90.

7 Izumi Y, Yamamoto T, Matsunaga N, Ota T, Owaki Y, Shinohara K, et al. Endometrial cancer arising from adenomyosis: case report and literature review of MRI findings. Radiol Case Rep. 2020;15:427-30. DOI: 10.1016/ j.radcr.2020.01.025, PMID: 32099587

8 Khalifa MA, Atri M, Klein ME, Ghatak S, Murugan P. Adenomyosis as a confounder to accurate endometrialc staging. Seminars in Ultrasound, CT and MRI. 2019;40:358-63. DOI: 10.1053/j.sult.2019.04.004, PMID: 31375175

9 Kaku T, Tsukamoto N, Tsuruchi N, Sugihara K, Kamura T, Nakano H. Endometrial metaplasia associated with endometrial carcinoma. Obstet Gynecol. 1992;80:812-6. PMID: 1407921

10 Kaku T, Silverberg SG, Tsukamoto N, Tsuruchi N, Kamura T, Saito $\mathrm{T}$, et al. Association of endometrial epithelial metaplasias with endometrial carcinoma and hyperplasia in Japanese and American women. Int J Gynecol Pathol. 1993;12:297-300. DOI: 10.1097/00004347-199310000-00003, PMID: 8253546 